\title{
The Effect of Diuretics on Patients with Heart Failure: A Network Meta- Analysis: Diuretics Effect on Heart Failure Patients
}

\author{
Xingsheng Zhao', MD; Yu Ren², MD; Hui Li ${ }^{1}$, MM; Xi Liu ${ }^{1}$, MD \\ ${ }^{1}$ Cardiology Department and ${ }^{2}$ Clinical Medical Research Center, Inner Mongolia People's Hospital, Hohhot, Inner \\ Mongolia Autonomous Region, China.
}

Received, April 10, 2019; Revised, May 6, 2019; Accepted, June 12 2019; Published, June 13, 2019.

\begin{abstract}
Purpose: We aimed to comprehensively evaluate the curative effect of torasemide, tolvaptan, furosemide and azosemide on patients with heart failure. Methods: Relevant studies were retrieved by searching the electronic databases until May 2018. Quality assessment and data extraction of selected studies were evaluated by two reviewers. Heterogeneity across studies was assessed utilizing the $\mathrm{I}^{2}$ statistic and Qtest, and appropriate effect model was selected to calculate the pooled effect size. Network meta-analysis was conducted and the convergence degree of model was evaluated. Results: A total of 12 studies were enrolled in this study. Significant heterogeneity was not identified across the studies. Significantly greater differences were found in left ventricular ejection fraction (LVEF) for furosemide VS. azosemide, in brain natriuretic peptide (BNP) for furosemide VS. azosemide and furosemide VS. torasemide, and in adverse effects for furosemide VS. torasemide through Meta-analysis of direct comparison. In addition, network meta-analysis results suggested there were no significant differences in adverse effects, mortality, BNP and LVEF among these groups. However, the relatively low mortality and small improvement of BNP and LVEF were found in HF patients treated with torasemide. Conclusion: Torasemide might be an optimal treatment for HF patients considering its comprehensive curative effect.
\end{abstract}

\section{INTRODUCTION}

Heart failure (HF) refers to the inability of the heart to provide the peripheral tissues with enough blood and oxygen to meet their metabolic demands [1]. It is estimated that $\mathrm{HF}$ affects about 40 million people globally in 2015 [2]. Approximately $1 \%$ to $2 \%$ of adult population is affected by $\mathrm{HF}$ [3]. HF prevalence increases with aging, from $6-10 \%$ in individuals aged $>65$ years to $>10 \%$ in individuals aged $>75$ years $[4,5]$. HF is related to reduced mental and physical health, leading to a significantly decreased quality of life [6, 7]. Therefore, it is essential to develop an optimal treatment for HF patients.

Diuretics, a mainstay of treatment for fluid accumulation, have been applied to treat HF [8], liver cirrhosis [9], hypertension [10], and certain kidney diseases [11]. It has been reported that loop diuretics restrain the reabsorption of water and sodium, and promote the urinary excretion of chloride, calcium, and magnesium; thus lead to a prompt diuretic effect [12]. Acute HF patients easily present the fluid retention, cardiac overload, and electrolyte disorders. Since diuretics relieve cardiac load, reduce bold pressure and fluid retention; hence, diuretic therapy is a crucial element in HF treatment [13-15]. The use of diuretics appeared to have improved mortality in HF individuals in small studies [16]. However, due to the small number of participants in the cited studies, it is unclear that the extent to which these results can be extrapolated to a general population [17]. Additionally, there are some adverse effects of diuretics during the treatment of HF, such as hypokalemia, hyponatremia, metabolic alkalosis, and hyperuricemia [18]. Therefore, both the efficacy and safety of diuretics should be considered during treating the HF patients.

Quick-acting diuretics torasemide and tolvaptan are often applied to treat chronic HF patients. Furosemide and azosemide are also the common diuretics for the treatment of $\mathrm{HF}$ and have certain therapeutic effect. But the comparison of efficacy and safety among these diuretics has not been elucidated decisively. In the present study, we aimed to comprehensively evaluate the therapeutic

Corresponding Authors: Xingsheng Zhao, Cardiology Department, E-mail: zhaoxingsh@hotmail.com; Yu Ren, Clinical Medical Research Cetre, Inner Mongolia People's Hospital, Zhao Wuda Road, Saipan District, Hohhot, Inner Mongolia Autonomous Region, China. 
effects of torasemide, tolvaptan, furosemide, and azosemide on HF patients through the network meta-analysis. This study is expected to obtain the optimal treatment for HF patients, and provide basis for the future clinical treatment.

\section{METHODS}

\section{Data sources}

Relevant literatures were obtained from the databases of Pubmed (http://www.ncbi.nlm.nih.gov/pubmed), Embase (http://www.embase.com), and Cochrane Library (http://www.cochranelibrary.com) until May 9, 2018. Search strategy was designed as follows: "Heart Failure" (OR "cardiac failure", OR "HF") AND "lasix" (OR "Torasemide", OR "furosemide", OR" Tolvaptan", OR "Azosemide").

\section{Inclusion and exclusion criteria}

Studies were included if they fulfilled with the following criteria: (1) public published metaanalysis studies about the diuretics effects on the treatment of patients with HF; (2) patients with HF undergone the treatment of diuretics in each group; (3) the outcome variables mainly included the incidence of postoperative adverse effects, postoperative mortality, left ventricular ejection fraction (LVEF), and brain natriuretic peptide (BNP); (4) the research types were randomized controlled trials (RCTs).

Meanwhile, studies were excluded if they met any of the following criteria: (1) the subjects were less than 10 in each group; (2) data were incomplete or cannot be used for statistical analysis; (2) studies were letters, reviews, or comments; (3) For the repeated publications or the same population data used in multiple literature, the latest or the most informative articles were included and the rest were excluded..

\section{Data extraction and quality assessment}

Relevant data was extracted by two independent reviewers from selected studies. The extraction contents contained the first author of study, publication year, survey area, research types, heart failure types, New York Heart Association (NYHA) stage, sample size in each group, demographic characteristics (such as age composition, sex ratio, pulse rate, body weight, and systolic blood pressure) in each group, the incidence of postoperative adverse effects, postoperative mortality, LVEF, and BNP. The quality assessment of RCT study was conducted utilizing the evaluation tool of bias risk recommended by the Cochrane Collaboration recommendations [19]. Any disagreements during the process of data extraction and quality assessment were resolved by discussion with the third investigator.

\section{STATISTICAL ANALYSIS}

Meta-analysis of direct comparison was performed utilizing the meta package in $\mathrm{R}$ (Version 3.12, Foundation for Statistical Computing, Beijing, China) software. The pooled effect size was represented with the mean difference (MD), odds ratio (OR) and $95 \%$ confidence intervals (CI) values [20]. Heterogeneity across studies was evaluated utilizing Q-test [21] and the $\mathrm{I}^{2}$ statistic. Significant heterogeneity was considered when $\mathrm{P}<$ 0.05 or $\mathrm{I}^{2}>50 \%$, and the random effects model was applied to calculate the pooled effect values. Otherwise, $\mathrm{P}>0.05$ and $\mathrm{I}^{2}<50 \%$ was considered non-heterogeneity, and the fixed effect model [22] was selected to calculate the effect size.

A network meta-analysis was performed utilizing the Aggregate Data Drug Information System (ADDIS, Version 1.16.5) software, which based on the Bayesian and Markov Chain Monte Carlo (MCMC) theories [23, 24]. All data were presented with the value of OR/MD and $95 \%$ CI. Besides, the random effects model was applied for all test models in the current study if there was not the closed loop for all studies. Moreover, the BrooksGelman-Rubin method was used to assess the convergence degree of model via the Potential Scale Reduction Factor (PSRF) indicator. Generally, the convergence of model was acceptable when PSRG values approached 1. It was also acceptable when PSRF values less than 1.2 [25].

\section{RESULTS}

\section{Characteristics of the inclusive studies}

Flow chart of literature retrieving was presented in Fig. 1. A total of 3,970 literatures were obtained from Pubmed (1,517 literatures), Embase (1,764 literatures), and Cochrane Library (689 literatures) databases. Among them, 1,027 duplicate literatures and 2589 obvious irrelevance studies were excluded, and a total of 354 literatures were remained. Then 307 literatures comprised of 27 letters, 16 case series/reports, 42 literature reviews, 222 no-relevant studies were excluded after scanning the abstract and title of literatures. Afterwards, 35 literatures were further excluded 
through reading the full-text, including 22 norelevant data and 13 non-RCT studies. Consequently, a total of 12 relevant studies were included in the present study for meta-analysis [2637], of these, 6 were multiple-center studies [26, 27, 30-33] and 4 single-center studies.

The characteristics of selected studies were summarized in Table 1. A total of 1,956 HF patients were included in the current study, including 271 azosemide-treated HF patients, 1005 furosemidetreated HF patients, 220 tolvaptan-treated HF patients, and 460 torasemide-treated HF patients. The publication year of selected studies was ranged from 1999 to 2017, and the survey areas contained Japan, United States, Spain, Switzerland and so on. HF types mainly included chronic HF, acute HF, and acute decompensated HF. No significant differences were found in male/female ratio, mean age, pulse rate, body weight, and systolic blood pressure in each group of all selected studies. In addition, RCT quality assessment revealed that all included literatures presented high quality, but performance bias and detection bias were observed in blinding of participants and personnel, and blinding of outcome assessment, respectively (Fig. 2).

Literature search in PubMed (1517), Embase (1764)

Cochanelibrary (689)

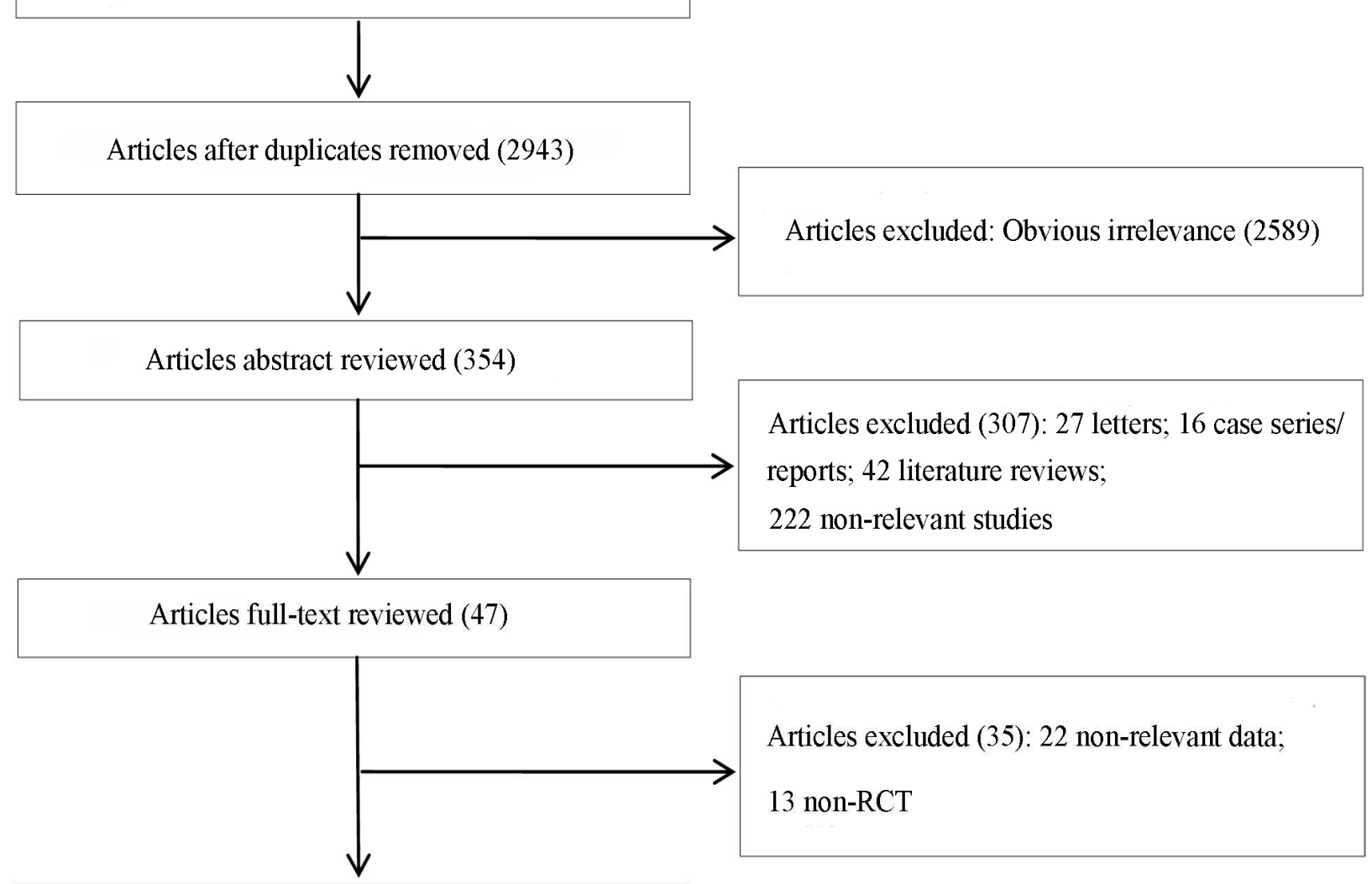

Articles included for Meta-analysis (12)

Figure 1. Flowchart of literature search and study selection. 


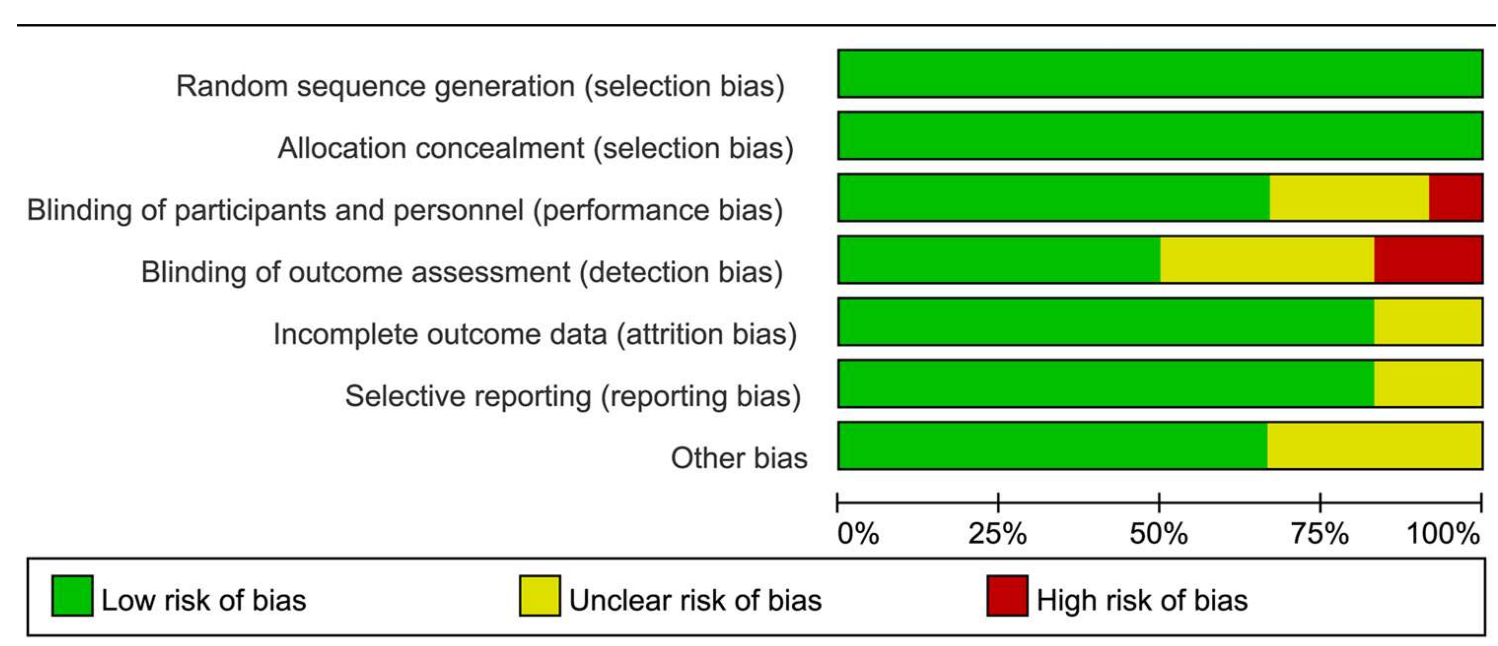

Figure 2. Aggregate included literatures risk-of-bias appraisal results. The green, yellow, and red colors represent the low, unclear and high risks of bias, respectively.

Results of Meta-analysis of direct comparison There was no significant heterogeneity identified across the studies $\left(P>0.05, \mathrm{I}^{2}=0\right)$, and the fixed effect model was utilized to calculate the pooled effect size. In terms of adverse effect, the incidence of torasemide group was significantly lower than furosemide group (OR $=0.66,95 \% \mathrm{CI}: 0.47-0.93)$, but not significant difference was observed for furosemide vs. tolvaptan and furosemide vs. azosemide groups (Fig. 3A). Similarly, no remarkable difference in the incidence of mortality was presented among patients treated with these diuretics (Fig. 3B). However, an obvious difference was identified in the BNP between furosemide and azosemide groups $(\mathrm{OR} / \mathrm{MD}=18.00,95 \% \mathrm{CI}$ : 8.4427.56), as well as difference between furosemide and torasemide groups $(\mathrm{OR} / \mathrm{MD}=57.55,95 \% \mathrm{CI}$ : 22.40 - 92.71) (Fig. 3C). Additionally, a significant difference was identified in the LVEF between furosemide and azosemide groups [OR/MD = $0.60,95 \%$ CI: $(-0.87)-(-0.32)]$, but no significant difference between was observed furosemide and torasemide groups [OR/MD $=-0.59,95 \% \mathrm{CI}$ : $(-$ 2.34) - (1.15)] (Fig.3D). These results preliminary indicated that no diuretics would simultaneously improve the incidence of adverse effects, mortality, LVEF, and BNP for HF patients.

\section{Results of Network meta-analysis}

The parameters for ADDIS software were set as follows: Number of chains: 4; Tuning iterations: 20000; Simulation iterations: 50000; Thinning interval: 10, Inference samples: 10000; Variance scaling factor: 2.5 . Then the network meta-analysis of the LVEF, BNP, the incidence of postoperative adverse effects, and postoperative mortality was performed.

The incidence of postoperative adverse effects For the incidence of postoperative adverse effects, all PSRF values were calculated between 1.00 and 1.01 , suggesting that the convergence of variables was complete with good iteration effect and stable results. Hence, the consistency model was applied for merging results. The results showed that HF patients treated with furosemide presented the lowest incidence of postoperative adverse effects on the basis of probabilities of ranking for each treatment in the network from the 1 st to the 4 th (Fig. 4A). The rank probabilities sum to one, both within a rank over all treatments and within a treatment over all ranks. Rank 1 is the worst indicating the highest incidence of adverse effects, and rank 4 is the best indicating the lowest incidence of adverse effects. Furosemide is better alternatives compared to the other treatments as they have much higher score on rank 4, which indicates they have much lower incidence of adverse effects. On the contrary, tolvaptan is the worst with highest rank 1 probability and lowest rank 4 probability. In general, the first, second, third, and fourth rank treatments were furosemide, azosemide, tolvaptan, and torasemide, respectively. However, no statistical significance among these groups was achieved as shown in Table 2, of which the results are the odds ratio with $95 \% \mathrm{CI}$ in the columndefining treatment compared with the row-defining treatment. 
A

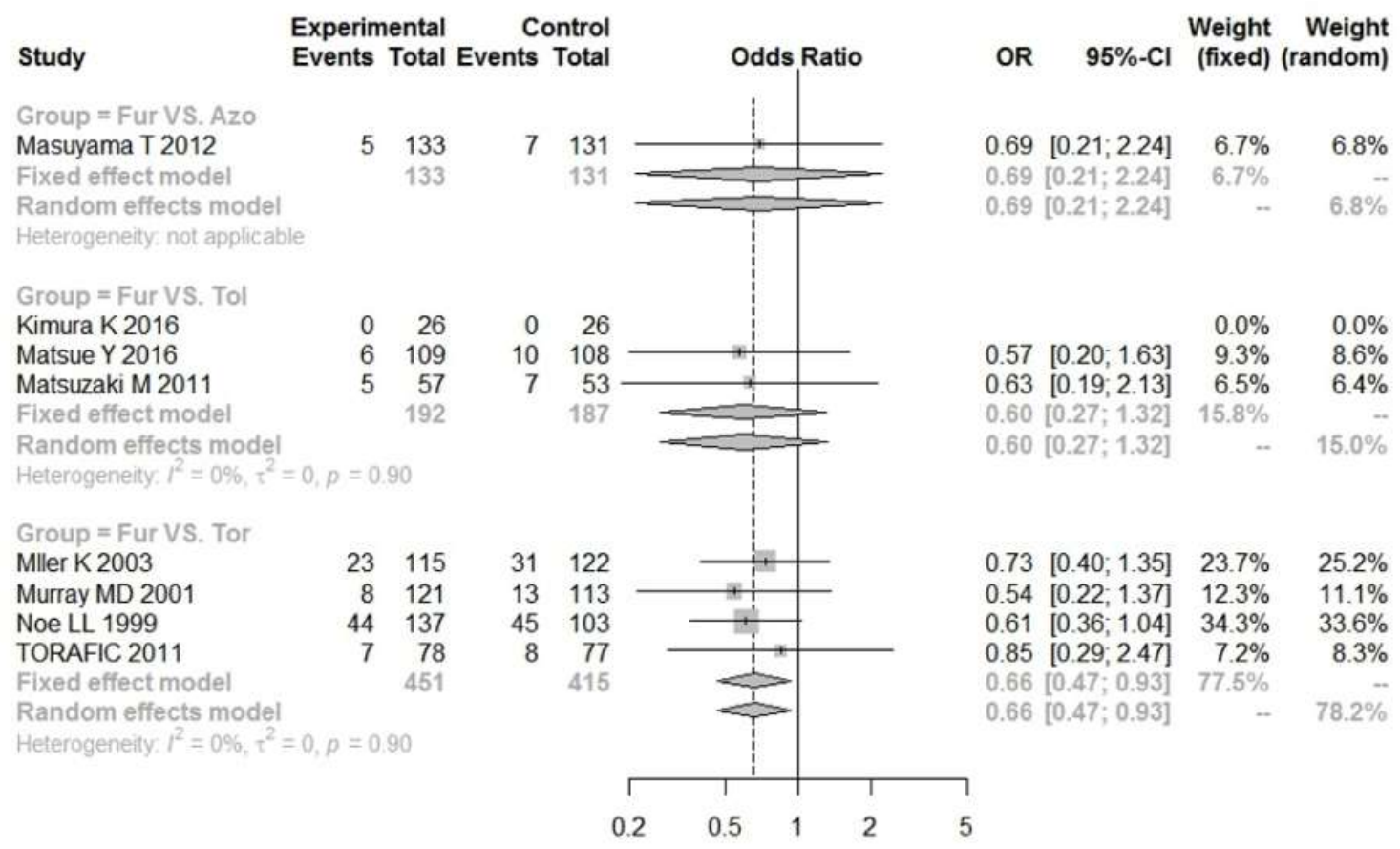

B

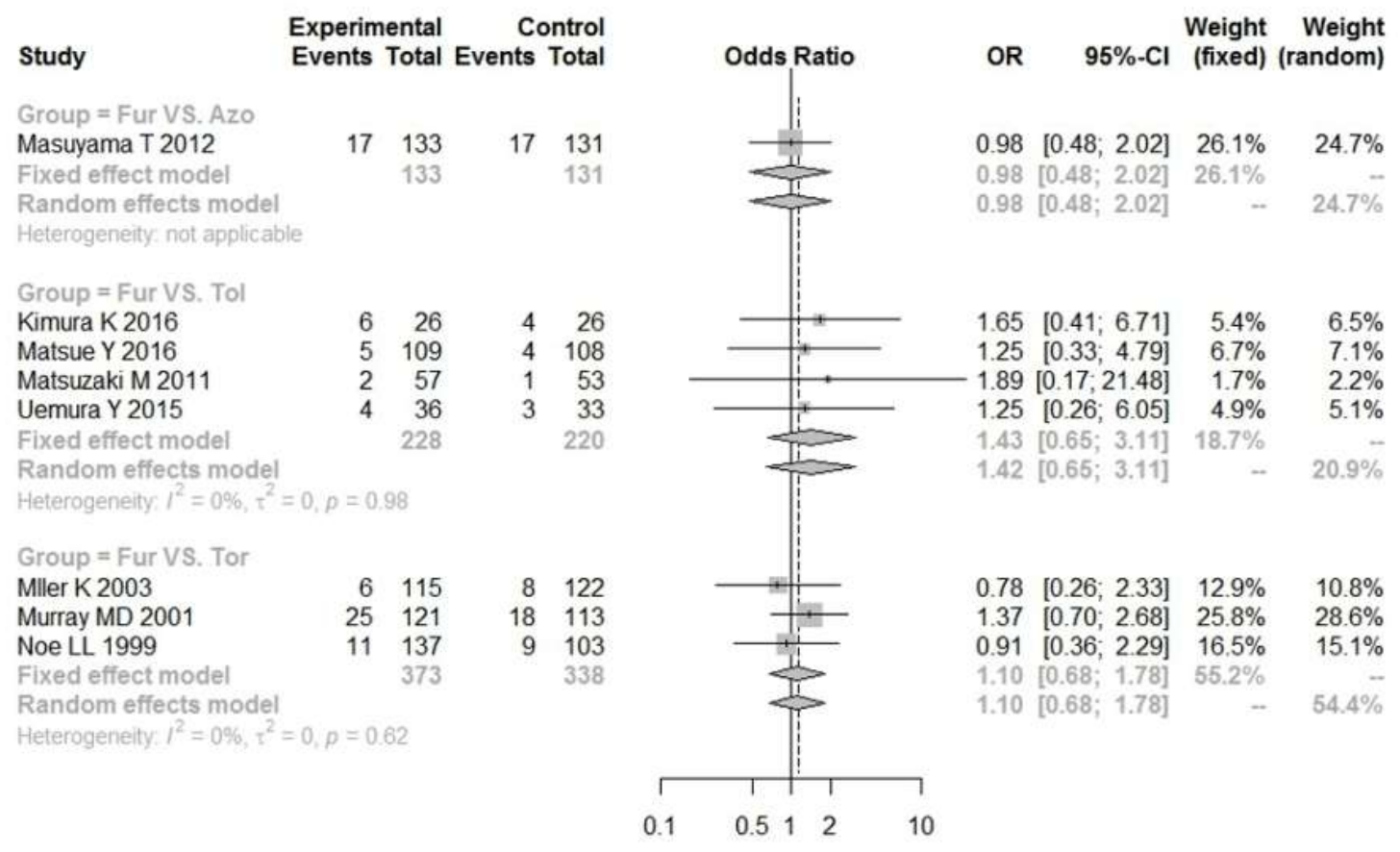

Figure 3 continued... 
C

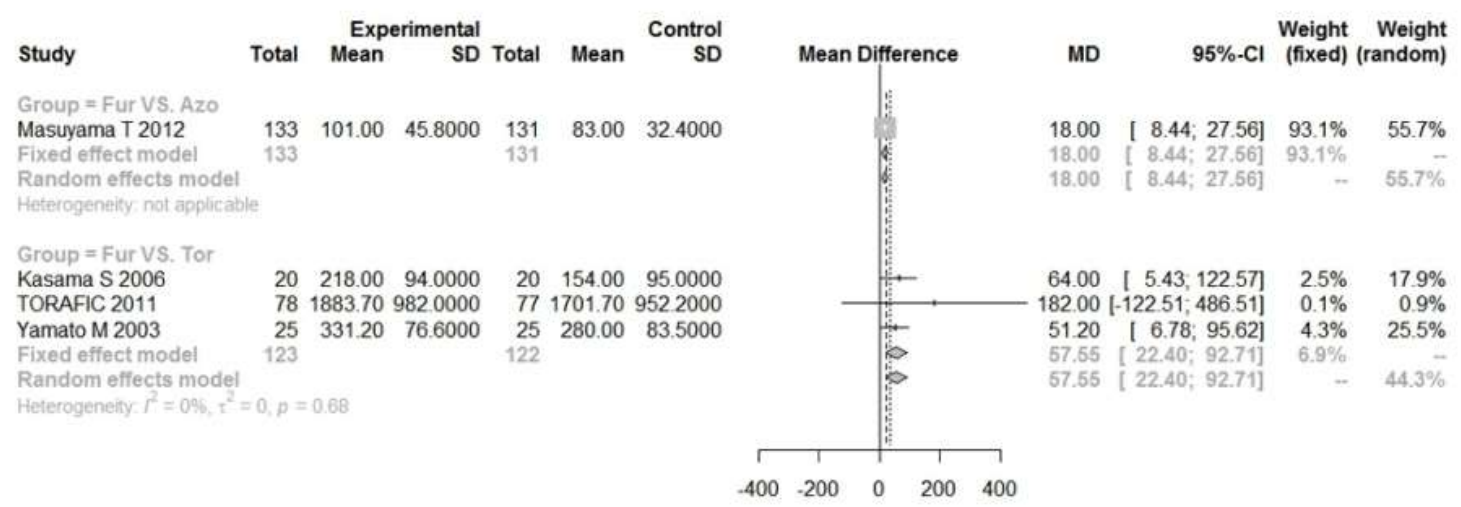

D

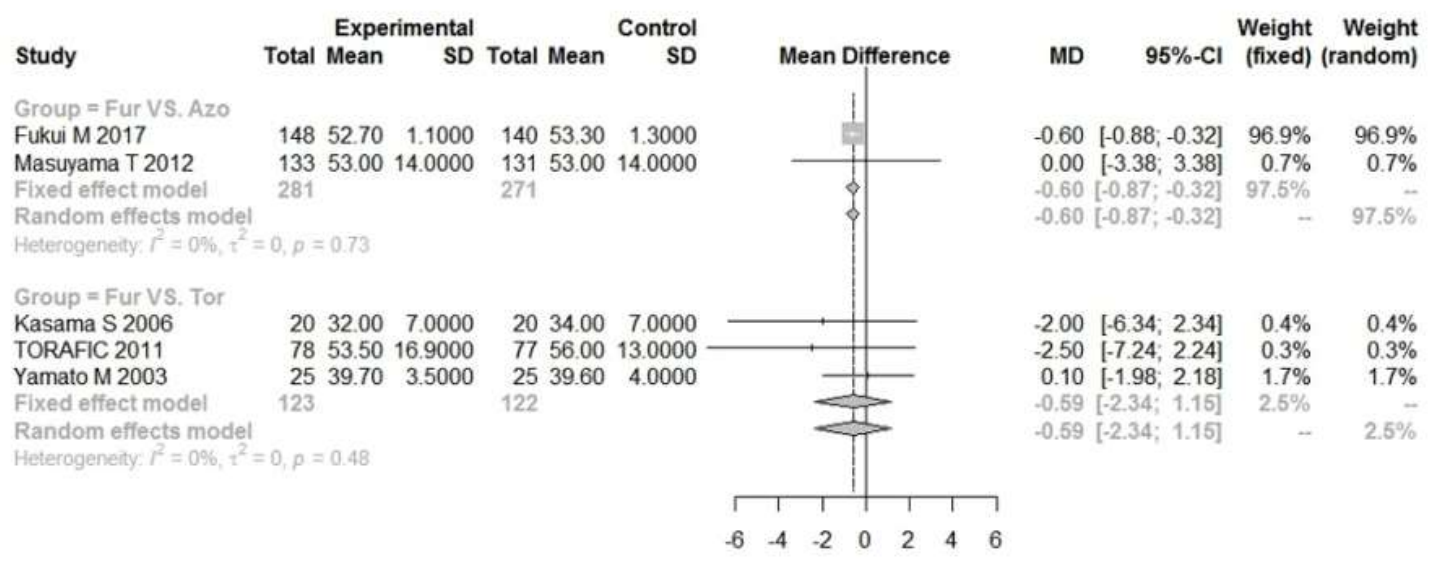

Figure 3. Forest plots from a meta-analysis of the incidence of adverse effects (A), postoperative mortality (B), brain natriuretic peptide (BNP, C), and left ventricular ejection fraction (LVEF, D). Squares represent point estimation and 95\% CI of random or fixed effect model. Horizontal lines represent point estimation and $95 \%$ CI of selected studies, in particular, the point in the horizontal lines represents the value of point estimation. Vertical solid lines stand for the standard line and vertical imaginary lines stand for the vertical axis of estimation value. OR: odds ratio; SD: standard deviation; MD: mean difference; CI: confidence interval.

Table 2 Network meta-analysis results of the incidence of postoperative adverse effects and postoperative mortality

\begin{tabular}{|c|c|c|c|}
\hline \multicolumn{4}{|c|}{ The incidence of postoperative adverse effects } \\
\hline Azosemide & $0.67(0.19,2.35)$ & $1.09(0.24,5.44)$ & $1.02(0.27,3.79)$ \\
\hline $1.50(0.43,5.18)$ & Furosemide & $1.66(0.71,4.21)$ & $1.52(0.97,2.36)$ \\
\hline $0.91(0.18,4.23)$ & $0.60(0.24,1.42)$ & Tolvaptan & $0.92(0.33,2.36)$ \\
\hline $0.98(0.26,3.69)$ & $0.66(0.42,1.03)$ & $1.09(0.42,3.04)$ & Torasemide \\
\hline \multicolumn{4}{|c|}{ Postoperative mortality } \\
\hline Azosemide & $0.99(0.42,2.29)$ & $0.63(0.20,2.18)$ & $0.92(0.31,2.54)$ \\
\hline $1.01(0.44,2.39)$ & Furosemide & $0.63(0.29,1.48)$ & $0.91(0.51,1.59)$ \\
\hline $1.59(0.46,5.00)$ & $1.58(0.68,3.43)$ & Tolvaptan & $1.45(0.50,3.76)$ \\
\hline $1.09(0.39,3.20)$ & $1.09(0.63,1.97)$ & $0.69(0.27,2.02)$ & Torasemide \\
\hline
\end{tabular}

Note. Results are the odds ratio with $95 \%$ CI in the column-defining treatment compared with the row-defining treatment. 
A

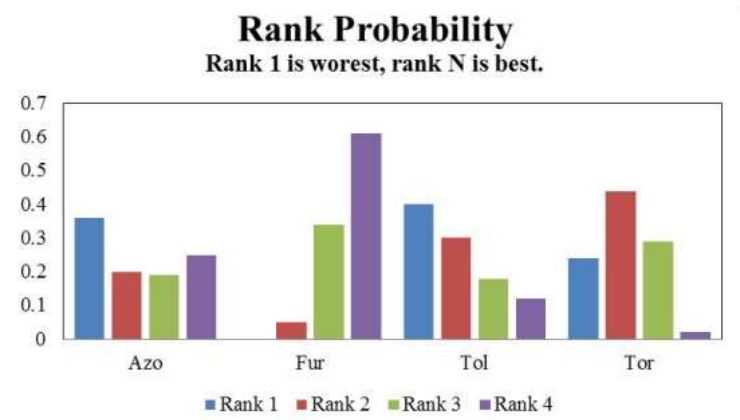

C

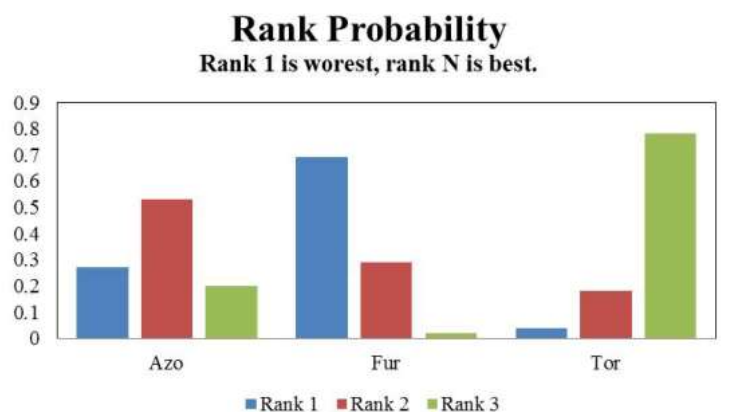

B

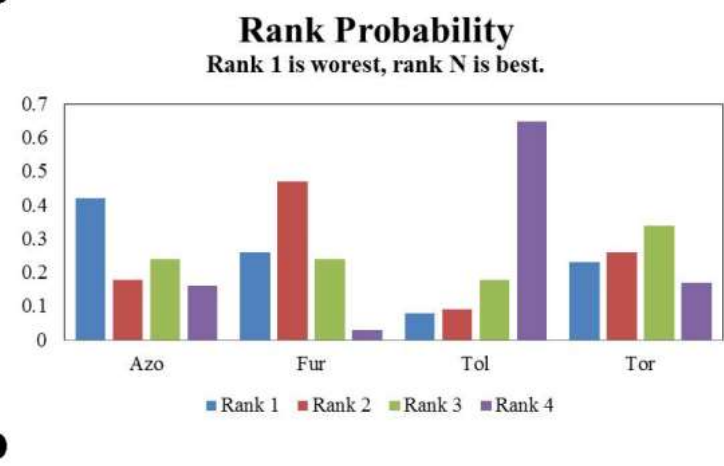

Rank Probability

Rank 1 is best, rank $\mathrm{N}$ is worest.

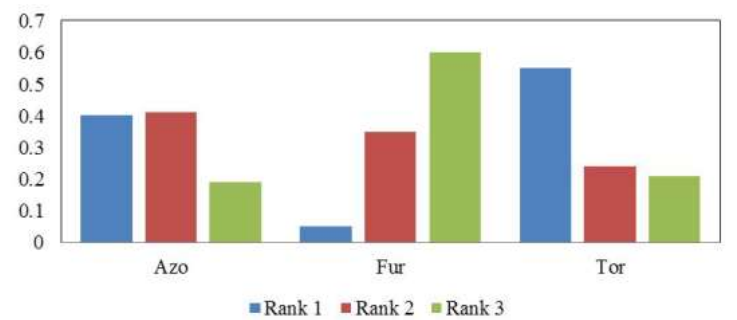

Figure 4. The rank probability plot for the incidence of adverse effects (A), postoperative mortality (B), BNP (C), and LVEF (D). The probability for each indicator to obtain each possible rank in terms of their relative effects (outcome value). The rank probabilities sum to one, both within a rank over all treatments and within a treatment over all ranks. Rank 1 stands for the worst curative effect for improvement of adverse effects, postoperative mortality and BNP among these diuretics treatments, while rank $\mathrm{N}$ (the largest number) stands for the best treatment. If the probability of rank 1 for one treatment group is highest, indicating this treatment had the worst curative effect for corresponding indicator. Whereas, the probability of rank $\mathrm{N}$ for one treatment group is highest, indicating this treatment had the best curative effect for corresponding indicator. In addition, for improvement of LVEF among these diuretics treatments, rank 1 stands for the best curative effect, while rank $\mathrm{N}$ (the largest number) stands for the worst treatment.

\section{Postoperative mortality}

For the postoperative mortality, all PSRF values were calculated between 1.00 and 1.01 , indicating that the convergence of variables was complete with good iteration effect and stable results. Hence, the consistency model was applied for merging results. Results showed that HF patients treated with tolvaptan presented the lowest mortality. The probabilities of ranking for each treatment in the network from the $1^{\text {st }}$ to the $4^{\text {th }}$ were shown in Fig. 4B. The first, second, third, and fourth rank treatments were tolvaptan, torasemide, azosemide, and furosemide, respectively. However, no statistical significance among groups was achieved (Table 2).

\section{LVEF and BNP levels}

For LVEF and BNP, all PSRF values were equal to 1.00 , indicating that the convergence of variables was complete with good iteration effect and stable results. Therefore, the consistency model was used to merge the results. The ranking results of each treatment probability in the network from the 1 st to the 3rd showed that the curative effect of torasemide on BNP and LVEF improvement was 1 st, next to azosemide (2st) and furosemide (3st), but only small improvement of BNP (Fig. 4C) and LVEF (Fig. 4D) with no statistic difference was found in torasemide group than in azosemide and furosemide groups (Table 3).

\section{DISCUSSION}

The results of this network meta-analysis showed that HF patients treated with furosemide presented the lowest incidence of postoperative adverse effects, but presented the highest mortality. Nevertheless, HF patients treated with tolvaptan presented a lower mortality compared to furosemide treatment. Importantly, HF patients treated with torasemide presented the small improvement of LVEF and BNP, and relatively low 
incidence of mortality, even though with the highest incidence of postoperative adverse effects. These results indicated that no diuretics would simultaneously improve the incidence of adverse effects, mortality, LVEF, and BNP for HF patients, while torasemide might be an optimal treatment for HF patients considering its comprehensive curative effect.

Previous study demonstrated that oral torasemide treatment significantly ameliorated the levels of LVEF and BNP in chronic HF patients compared with oral furosemide treatment [37]. Our results showed small but not statistically significant improvement of BNP and LVEF levels were found in HF patients treated with torasemide than treated with other azosemide and furosemide, according to the probabilities of ranking for each treatment in the network meta-analysis. The increased natriuretic peptides are detected in most types of heart failure [38]. In addition, reduced LVEF as a key selection criterion for heart failure trials and the importance of assessing LVEF is also a quality indicator in the management of HF [39]. Previous study found that torasemide also obviously improved cardiac function to reduce chronic $\mathrm{HF}$ and arrhythmia [40]. Several studies uncovered that the torasemide treatment in NYHA class in patients with congestive chronic HF was superior to furosemide treatment [33, 34, 41]. However, our results suggested that HF patients treated with torasemide presented the highest incidence of postoperative adverse effects in the network metaanalysis, which was not consistent with some previous studies. For instance, torasemide effectively ameliorate BNP levels, HF symptoms and signs for $\mathrm{HF}$ in children, without any significant adverse effects [42]. We speculated that the incomplete demographic characteristics data of the selected studies might affect the reliability of our results. In addition, there is likely to present a bias for the results of adverse effects because it is an overall result for the incidence of adverse effects. Therefore, more studies were required for consolidating the result of adverse effect in the future. In particular, the postoperative mortality in HF patients treated with torasemide was lower than HF patients treated with furosemide and azosemide treatments. Generally, the adverse effects can be controlled in advance, thus mortality is a more serious outcome than the incidence of adverse effects. Therefore, we concluded that the torasemide treatment with the higher efficacy and lower postoperative mortality in HF patients was likely to superior to other treatments.

With regarding to other diuretics, our results showed that the BNP level was dramatically ameliorated in HF patients treated with azosemide compared with furosemide treatment. The results were consistent with Fukui et al's study that azosemide effectively improved the BNP levels in chronic HF patients [26]. It was reported that azosemide reduced the risk of cardiovascular death for congestive HF compared with furosemide [30]. Furosemide may be associated with the increased risk for cardiovascular mortality, and has adverse effect on patients [43]. Consistent with previous study, our results indicated that HF patients presented the higher mortality after treatment with furosemide compared with azosemide treatment. Furthermore, Uemura and his colleagues demonstrated that tolvaptan treatment reduced the risk of worsening renal function for acute decompensated HF patients with severe chronic kidney disease [36]. Besides, our results indicated that the incidence of adverse effects of tolvaptan treatment on HF patients was lower than torasemide treatment. These finding suggested that diuretics mentioned above wouldn't simultaneously improve the incidence of adverse effects, mortality, LVEF, and BNP for HF patients, and more updating studies were need for further analysis.

Table 3 Network meta-analysis results of LVEF and BNP

\begin{tabular}{lll}
\hline LVEF & & \\
\hline Azosemide & $-0.56(-2.60,1.82)$ & $0.29(-2.62,3.85)$ \\
$0.56(-1.82,2.60)$ & Furosemide & $0.85(-1.40,3.37)$ \\
$-0.29(-3.85,2.62)$ & $-0.85(-3.37,1.40)$ & Torasemide \\
BNP & & $-39.24(-241.99,134.60)$ \\
Azosemide & $18.73(-136.21,189.89)$ & $-60.22(-186.16,27.07)$ \\
$-18.73(-189.89,136.21)$ & Furosemide & Torasemide \\
$39.24(-134.60,241.99)$ & $60.22(-27.07,186.16)$ & \\
\hline Note. Results are the odds ratio with 95\% CI in the column-defining treatment compared with the row-defining \\
treatment. LVEF, left ventricular ejection fraction; BNP, brain natriuretic protein.
\end{tabular}


There are still some limitations in this study. First, the results failed to be adjusted by concomitant variables because of some incomplete data of studies, which might be as potential confounders to influence the pooled results. Additionally, subgroup analyses were not performed because of the incomplete data. Moreover, the reported results can be limited as ADDIS software cannot be freely programmed. For instance, only the random effect model can be reported during the estimate of effect size. Hence, the results of our study may be estimated conservatively. Finally, the network meta-analysis for other important indicators was not carried out due to lacking the proper literatures, and only four indicators were analyzed in this network metaanalysis.

In summary, this is the first network metaanalysis to analyze the efficacy of torasemide, tolvaptan, furosemide, and azosemide on $\mathrm{HF}$ patients. In particular, our study revealed that torasemide may be an optimal treatment for $\mathrm{HF}$ patients.

\section{COMPETING INTERESTS}

All authors declare that they have no competing interest to state.

\section{REFERENCES}

1. Tanai, E. and S. Frantz, Pathophysiology of Heart Failure. Compr Physiol, 2015. 6(1): p. 187-214.

2. Global, regional, and national incidence, prevalence, and years lived with disability for 310 diseases and injuries, 1990-2015: a systematic analysis for the Global Burden of Disease Study 2015. Lancet, 2016. 388(10053): p. 1545-1602.

3. Metra, M. and J. Teerlink, Heart failure. Lancet, 2017. 390(10106): p. 1981-1995.

4. McMurray, J. and M. Pfeffer, Heart failure. Lancet, 2005. 365(9474): p. 1877-89.

5. Dickstein, K., et al., ESC Guidelines for the diagnosis and treatment of acute and chronic heart failure 2008: the Task Force for the Diagnosis and Treatment of Acute and Chronic Heart Failure 2008 of the European Society of Cardiology. Developed in collaboration with the Heart Failure Association of the ESC (HFA) and endorsed by the European Society of Intensive Care Medicine (ESICM). Eur. Heart J., 2008. 29(19): p. 2388-442.

6. Juenger, J., et al., Health related quality of life in patients with congestive heart failure: comparison with other chronic diseases and relation to functional variables. Heart, 2002. 87(3): p. 235-41.

7. Hobbs, F., et al., Impact of heart failure and left ventricular systolic dysfunction on quality of life: a cross-sectional study comparing common chronic cardiac and medical disorders and a representative adult population. Eur. Heart J., 2002. 23(23): p. 1867-76.

8. Krishnamoorthy, A. and G. Felker, Fluid removal in acute heart failure: diuretics versus devices. Curr Opin Crit Care, 2014. 20(5): p. 478-83.

9. Chiba, M., et al., Diuretics aggravate zinc deficiency in patients with liver cirrhosis by increasing zinc excretion in urine. Hepatology Research, 2013. 43(4): p. 365.

10. Ernst, M.E. and M. Moser, Use of diuretics in patients with hypertension. N Engl J Med, 2009. 361(22): p. 2153.

11. Vasavada, N., C. Saha, and R. Agarwal, A doubleblind randomized crossover trial of two loop diuretics in chronic kidney disease. Kidney International, 2003. 64(2): p. 632-640.

12. Hutcheon, D.E., D. Mehta, and A. Romano, DIURETIC ACTION OF FUROSEMIDE. Archives of Internal Medicine, 1965. 115(5): p. 542.

13. Islam, M.S., The Art and Science of Using Diuretics in the Treatment of Heart Failure in Diverse Clinical Settings. Adv Exp Med Biol, 2018. 1067: p. 47-65.

14. Koratala, A., Metabolic abnormalities in heart failure patients treated with ultrafiltration versus diuretics. Eur J Heart Fail, 2018.

15. Yamazoe, M., et al., Incidence of hospital-acquired hyponatremia by the dose and type of diuretics among patients with acute heart failure and its association with long-term outcomes. J Cardiol, 2018. 71(6): p. 550-556.

16. Faris, R., et al., Diuretics for heart failure. Cochrane Database Syst Rev, 2012: p. CD003838.

17. von Lueder, T., D. Atar, and H. Krum, Diuretic use in heart failure and outcomes. Clin. Pharmacol. Ther., 2013. 94(4): p. 490-8.

18. Boron, W.F. and E.L. Boulpaep, Medical Physiology: A Cellular and Molecular Approach. 2009.

19. Higgins, J.P. and S. Green, Cochrane Handbook for Systematic Reviews of Interventions: Cochrane Book Series. 2008: Wiley-Blackwell. 102-8.

20. Liu, T., et al., Occupational exposure to methylene chloride and risk of cancer: a meta-analysis. Cancer Causes \& Control, 2013. 24(12): p. 2037-2049.

21. Lau, J., J.P. Ioannidis, and C.H. Schmid, Quantitative synthesis in systematic reviews. Ann Intern Med, 1997. 127(9): p. 820-6.

22. Feng, R.N., et al., Meta-Analysis ofTNF308 G/A Polymorphism and Type 2 Diabetes Mellitus. PLoS One, 2011. 6(4): p. e18480.

23. Hillege, H., et al., ADDIS: an automated way to do network meta-analysis. 2012, University of Groningen, Research Institute SOM (Systems, Organisations and Management).

24. Van Valkenhoef, G., et al., ADDIS: a decision support system for evidence-based medicine. 
Decision Support Systems, 2013. 55(2): p. 459-475.

25. Brooks, S.P. and A. Gelman, General methods for monitoring convergence of iterative simulations. Journal of computational and graphical statistics, 1998. 7(4): p. 434-455.

26. Fukui, M., et al., Changes in brain natriuretic peptide in chronic heart failure patients treated with long-acting versus short-acting loop diuretics: JMELODIC subanalysis. Heart Vessels, 2017. 32(7): p. $865-871$.

27. Group, T.I., Effects of prolonged-release torasemide versus furosemide on myocardial fibrosis in hypertensive patients with chronic heart failure: a randomized, blinded-end point, active-controlled study. Clin Ther, 2011. 33(9): p. 1204-1213 e3.

28. Kasama, S., et al., Effects of torasemide on cardiac sympathetic nerve activity and left ventricular remodelling in patients with congestive heart failure. Heart, 2006. 92(10): p. 1434-40.

29. Kimura, K., et al., Early administration of tolvaptan preserves renal function in elderly patients with acute decompensated heart failure. J Cardiol, 2016. 67(5): p. 399-405.

30. Masuyama, T., et al., Superiority of Long-Acting to Short-Acting Loop Diuretics in the Treatment of Congestive Heart Failure. Circulation Journal, 2012. 76(4): p. 833-842.

31. Matsue, Y., et al., Clinical Effectiveness of Tolvaptan in Patients With Acute Heart Failure and Renal Dysfunction. J Card Fail, 2016. 22(6): p. 42332.

32. Matsuzaki, M., et al., Efficacy and safety of tolvaptan in heart failure patients with volume overload despite the standard treatment with conventional diuretics: a phase III, randomized, double-blind, placebo-controlled study (QUEST study). Cardiovasc Drugs Ther, 2011. 25 Suppl 1: p. S33-45.

33. Müller, K., et al., Torasemide vs. furosemide in primary care patients with chronic heart failure NYHA II to IV-efficacy and quality of life. European Journal of Heart Failure, 2003. 5(6): p. 793-801.

34. Murray, M.D., et al., Open-label randomized trial of torsemide compared with furosemide therapy for patients with heart failure. American Journal of Medicine, 2001. 111(7): p. 513-520.

35. Noe, L.L., et al., A pharmacoeconomic assessment of torsemide and furosemide in the treatment of patients with congestive heart failure. Clin Ther, 1999. 21(5): p. 854-66.

36. Uemura, Y., et al., Clinical benefit of tolvaptan in patients with acute decompensated heart failure and chronic kidney disease. Heart Vessels, 2016.31(10): p. 1643-9.

37. Yamato, M., et al., Effects of torasemide on left ventricular function and neurohumoral factors in patients with chronic heart failure. Circulation Journal, 2003. 67(5): p. 384-390.
38. Cleland, J.G. and A.L. Clark, Heart failure - does it matter whether LVEF is reduced? Lancet, 2012. 380(9851): p. 1363-1365.

39. Ajello, L., et al., Diagnosis and treatment of asymptomatic left ventricular systolic dysfunction after myocardial infarction. Isrn Cardiology, 2015. 2013(3): p. 731285.

40. Han, L., et al., Torasemide reduces dilated cardiomyopathy, complication of arrhythmia, and progression to heart failure. Genet. Mol. Res., 2014. 13(3): p. 7262-74.

41. Spannheimer, A., A. Goertz, and B. DreckmannBehrendt, Comparison of therapies with torasemide or furosemide in patients with congestive heart failure from a pharmacoeconomic viewpoint. International Journal of Clinical Practice, 1998. 52(7): p. 467.

42. Senzaki, H., et al., Efficacy and safety of torasemide in children with heart failure. Arch. Dis. Child., 2008. 93(9): p. 768-71.

43. Domanski, M., et al., Diuretic use, progressive heart failure, and death in patients in the Studies Of Left Ventricular Dysfunction (SOLVD). Journal of the American College of Cardiology, 2003. 42(4): p. 705-708. 
J Pharm Pharm Sci (www.cspsCanada.org) 22, 270 - 280, 2019

Table 1 Characteristics of included studies

\begin{tabular}{|c|c|c|c|c|c|c|c|c|c|c|c|c|c|c|c|c|}
\hline Author & Year & Location & $\mathrm{MC} / \mathrm{SC}$ & Type & $\begin{array}{c}\text { NYHA } \\
\text { class }\end{array}$ & Group & $\mathrm{N}$ & Age (Mean \pm SD) & $\begin{array}{c}\text { Male/ } \\
\text { Female }\end{array}$ & $\begin{array}{c}\text { Pulse rate } \\
\text { (beatsy/min) }\end{array}$ & Body weight (kg) & $\mathrm{SBP}(\mathrm{mmHg})$ & $\mathrm{M}$ & LVEF & BNP & Adverse Effect \\
\hline \multirow{2}{*}{ Fukui M } & \multirow{2}{*}{2017} & \multirow{2}{*}{ Japan } & \multirow{2}{*}{$\mathrm{MC}$} & \multirow{2}{*}{$\mathrm{CHF}$} & \multirow{2}{*}{ II, III } & Fur & 148 & $71 \pm 10$ & $95 / 58$ & $71 \pm 13$ & $59 \pm 12$ & $124 \pm 16$ & NA & $52.7 \pm 1.1$ & $4.60 \pm 0.09 *$ & NA \\
\hline & & & & & & Azo & 140 & $71 \pm 11$ & $87 / 53$ & $72 \pm 13$ & $60 \pm 11$ & $127 \pm 17$ & NA & $53.3 \pm 1.3$ & $4.26 \pm 0.09^{*}$ & NA \\
\hline \multirow{2}{*}{ Kasama S } & \multirow{2}{*}{2006} & \multirow{2}{*}{ Japan } & \multirow{2}{*}{$\mathrm{SC}$} & \multirow{2}{*}{$\begin{array}{l}\text { Non- } \\
\text { ischaemic } \\
\text { CHF }\end{array}$} & \multirow{2}{*}{ II, III } & Tor & 20 & $68 \pm 6$ & $15 / 5$ & NA & $58 \pm 9$ & $133 \pm 15$ & NA & $34 \pm 7$ & $154 \pm 95$ & NA \\
\hline & & & & & & Fur & 20 & $68 \pm 9$ & $14 / 6$ & NA & $59 \pm 10$ & $131 \pm 16$ & NA & $32 \pm 7$ & $218 \pm 94$ & NA \\
\hline \multirow{2}{*}{ Kimura K } & \multirow{2}{*}{2016} & \multirow{2}{*}{ Japan } & \multirow{2}{*}{$\mathrm{SC}$} & \multirow{2}{*}{$\mathrm{ADHF}$} & \multirow{2}{*}{ III,IV } & Tol & 26 & $80.54 \pm 12.15$ & $10 / 16$ & $87.5 \pm 20.3$ & $56.32 \pm 14.80$ & $147.5 \pm 18.0$ & 4 & NA & NA & 0 \\
\hline & & & & & & Fur & 26 & $86.15 \pm 4.95$ & $12 / 14$ & $79.2 \pm 23.8$ & $51.95 \pm 12.05$ & $142.0 \pm 25.2$ & 6 & NA & NA & 0 \\
\hline \multirow{2}{*}{$\begin{array}{c}\text { Masuyama } \\
\mathrm{T}\end{array}$} & \multirow{2}{*}{2012} & \multirow{2}{*}{ Japan } & \multirow{2}{*}{$\mathrm{MC}$} & \multirow{2}{*}{$\mathrm{CHF}$} & \multirow{2}{*}{ II, III } & Fur & 133 & $71 \pm 11$ & $68 / 65$ & $71 \pm 14$ & $59.0 \pm 12.8$ & $125 \pm 17$ & 17 & $53 \pm 14$ & $101 \pm 45.8$ & 5 \\
\hline & & & & & & Azo & 131 & $71 \pm 11$ & $72 / 59$ & $72 \pm 13$ & $59.8 \pm 13.3$ & $126 \pm 18$ & 17 & $53 \pm 14$ & $83 \pm 32.4$ & 7 \\
\hline \multirow{2}{*}{ Matsue Y } & \multirow{2}{*}{2016} & \multirow{2}{*}{ Japan } & \multirow{2}{*}{$\mathrm{MC}$} & $\mathrm{AHF}$ & IIIJV & Tol & 108 & $72.99 \pm 8.90$ & $72 / 36$ & $94.2 \pm 27.3$ & NA & $145.8 \pm 32.9$ & 4 & NA & $\mathrm{NA}$ & 10 \\
\hline & & & & & & Fur & 109 & $72.95 \pm 10.24$ & $69 / 40$ & $88.6 \pm 23.4$ & NA & $142.1 \pm 28.1$ & 5 & NA & NA & 6 \\
\hline Matsuzaki & 2011 & Janan & $\mathrm{MC}$ & $\mathrm{HF}$ & IJI JIJV & Tol & 53 & $71.3 \pm 10.6$ & $35 / 18$ & NA & $60.9 \pm 13.0$ & NA & 1 & NA & $\mathrm{NA}$ & 7 \\
\hline M & & & & & & Fur & 57 & $71.0 \pm 10.9$ & $39 / 18$ & NA & $56.8 \pm 13.0$ & NA & 2 & NA & NA & 5 \\
\hline Muler K & 2003 & Switzerlan & $\mathrm{MC}$ & CHF & UI UU IV & Tor & 122 & $74.4 \pm 11.0$ & $55 / 67$ & $79.3 \pm 15.8$ & $77.9 \pm 17.1$ & $141.7 \pm 21.3$ & 8 & NA & NA & 31 \\
\hline viller K & 2003 & & $\mathrm{MC}$ & CHF & II, III,IV & Fur & 115 & $73.2 \pm 10.2$ & $47 / 68$ & $76.5 \pm 11.3$ & $76.2 \pm 18.7$ & $142.8 \pm 24.0$ & 6 & NA & NA & 23 \\
\hline Murray MD & 2001 & USA & $\mathrm{NA}$ & $\mathrm{CHF}$ & NA & Fur & 121 & $64.1 \pm 12.4$ & $56 / 65$ & NA & $84.6 \pm 22.0$ & $137 \pm 26$ & 25 & NA & NA & 8 \\
\hline & & & & & & Tor & 113 & $64.1 \pm 10.9$ & $55 / 58$ & NA & $85.9 \pm 23.0$ & $133 \pm 27$ & 18 & NA & NA & 13 \\
\hline Noe LL & 1999 & USA & $\mathrm{SC}$ & $\mathrm{CHF}$ & II, III & Tor & 103 & Mean:75.1 & $59 / 44$ & NA & Mean:180IB & NA & 9 & NA & NA & 45 \\
\hline 1 VUC LL & צערו & - & SC & C111 & & Fur & 137 & Mean:75.1 & $74 / 63$ & NA & Mean:163IB & NA & 11 & NA & NA & 44 \\
\hline TORAFIC & 2011 & Snain & $\mathrm{MC}$ & CHF & UI II & Tor & 77 & $68.1 \pm 11.4$ & $42 / 35$ & $68.6 \pm 12.8$ & $82.3 \pm 15.5$ & $133.5 \pm 18.2$ & NA & $56.0 \pm 13.0$ & $170.2 \pm 95.2$ & 8 \\
\hline IUKAFIC & 2011 & spain & $\mathrm{MC}$ & CHF & II, III & Fur & 78 & $69.3 \pm 9.8$ & $48 / 30$ & $69.8 \pm 13.3$ & $80.4 \pm 15.8$ & $137.6 \pm 19.1$ & NA & $53.5 \pm 16.9$ & $188.4 \pm 98.2$ & 7 \\
\hline Uemura Y & 2015 & Janan & $\mathrm{SC}$ & $\mathrm{ADHF}$ & III IV & Tol & 33 & $75.1 \pm 12.3$ & $15 / 18$ & NA & NA & $134.1 \pm 31.6$ & 3 & NA & NA & NA \\
\hline Uemura $r$ & & Japan & $\mathrm{SC}$ & АDПГ & III,IV & Fur & 36 & $79.4 \pm 9.9$ & $19 / 17$ & $\mathrm{NA}$ & NA & $135.0 \pm 39.2$ & 4 & $\mathrm{NA}$ & NA & NA \\
\hline Yamato $\mathrm{M}$ & 2003 & Janan & NA & CHF & UI UU & Fur & 25 & $64.9 \pm 6.2$ & $14 / 11$ & $71.8 \pm 11.5$ & $61.4 \pm 10.9$ & $120.0 \pm 10.1$ & $\mathrm{NA}$ & $39.7 \pm 3.5$ & $331.2 \pm 76.6$ & NA \\
\hline Yamato M & 2003 & Japan & NA & $\mathrm{CHF}$ & 11, 111 & Tor & 25 & $64.7 \pm 6.1$ & $14 / 11$ & $70.2 \pm 10.7$ & $60.4 \pm 11.8$ & $123.6 \pm 10.7$ & NA & $39.6 \pm 4.0$ & $280.0 \pm 83.5$ & NA \\
\hline
\end{tabular}

Note. Fur, Furosemide; Tol, Tolvaptan; Azo, Azosemide; Tor, Torasemide; BNP, brain natriuretic protein; *: LnBNP; M, mortality; MC, multicenter; SC, single center; NYHA, New

York Heart Association; ADHF, acute decompensated heart failure; AHF, Acute Heart Failure; CHF, chronic heart failure; SBP, Systolic blood pressure; LVEF, left ventricular ejection fraction. 